UDC 614.1

DOI: 10.21668/health.risk/2016.4.07.eng

\title{
PECULIARITIES OF TERRITORIAL DISTRIBUTION AND DYNAMICS IN RATES OF POPULATION NONCOMMUNICABLE DISEASES IN THE KRASNOYARSK REGION ASSOCIATED WITH THE INFLUENCE OF ENVIRONMENTAL RISK FACTORS
}

\author{
D.V. Goryaev, I.V. Tikhonova \\ Administration of the Federal Supervision Service for Consumer's Rights Protection and Human Welfare \\ in the Krasnoyarsk Region, 21 Karatanova Str., Krasnoyarsk, 660049, Russian Federation
}

The analysis of the primary morbidity dynamics in the population of the Krasnoyarsk region is performed for the period 2005-2015. The incidence forecast by classes of ICD diseases, X revision is calculated. It was found that the figures of the first time revealed incidence in the Krasnoyarsk region exceeded the average for the Russian Federation during the years 2011-2014 on neoplasms, nervous system diseases, diseases of the circulatory and the digestive systems, diseases of the musculoskeletal system and connective tissue, endocrine diseases and a number of other systems. The values of the integral health risk indicators are calculated by the methodology of State Sanitary and Epidemiological Surveillance Agency of the year 1995. For risk characteristics the following criteria are used: integral index of health less than <0.312 - minimal risk; 0.313-0.500 - moderate risk; 0.501-0.688 - increased risk of more than 0.689- the highest risk. It was found that the increased health risk is typical of 21 municipal territories of the region, which is home to $66.1 \%$ of the population. Among the areas with "high" public health risk in different years were Sharypovo, Birilyussy and Evenk districts. In most areas the priorities relate to a group of non-communicable diseases associated with the negative impact of various factors of habitat population (disease of the respiratory system, circulatory system, neoplasms, including malignant, etc.). According to the forecasts in 2016 we expect the decrease in the proportion of inhabitants in the region from the group with the "moderate" and "elevated" risk, and an increase in the number of people with "high" and "minimal" risk to public health. It is shown that a number of regional municipalities have a necessity in the planning and implementation of measures to improve the population health.

Key words: Krasnoyarsk region, primary morbidity, integrated health assessment, risk areas.

Mineral resources extraction, processing industries, and electricity, gas and water supply develop in the Russian Federation, and production volumes in these spheres have already been growing for a long time. It all leads to greater anthropogenic load on the environment.

Environment contamination caused by various production branches, as well as occurrence of new challenges and threats, damages population health and it is an acute ecologic problem having great social and economic significance
$[9,11,14]$.

Industrial production is concentrated in big cities and most people also live there so ecologic situation in them is rather tense and it is connected both with current enterprises functioning and ecological problems inherited from past activities [16].

Unfavorable environment existing in contemporary cities and being caused by intense contaminating emissions of industrial enterprises and transport leads to growth in morbidity and

(C) Goryaev D.V., Tikhonova I.V., 2016

Dmitry V. Goryaev - director, chief state sanitary doctor of the Krasnoyarsk Region (e-mail: goryaev_dv@24.rospotrebnadzor.ru; tel.: +7 (391) 226-89-50).

Irina V. Tikhonova - Head of social and hygienic monitoring (e-mail: Tihonova_iv@24.rospotrebnadzor.ru; tel.: + 7 (391) 226-89-91). 
occurrence of diseases in all population groups, first of all children; morbidity in its turn results in economic losses of the state $[2,4-7,10,13,15]$.

It becomes quite evident that population health is an integrated index of human environment quality and its influence on life activity of a human body. Thereby public health indexes become integral indexes of medical and ecological welfare and can be used as criteria for its assessment; ecological processes can therefore be considered top determinants of human welfare $[1,3,12,17]$.

Krasnoyarsk region can be characterized as having highly developed industry and growing contamination levels caused by motor transport. Industries on this territory exert significant influence on human environment. And here we note that population health monitoring becomes really essential as it provides data for examining influence environmental factors have on population health. It helps to create priority measures aimed at eliminating adverse effects caused by these factors as well as to assess population health and to give forecasts on it. Population health is a whole set of quantitative indexes where morbidity indexes are among the most important ones. And if we take Krasnoyarsk region we can see that average morbidity indexes for many diseases categories here constantly exceed average levels in the RF and such trends are characteristic for population health in this region.

Research goal: to assess primary morbidity levels and dynamics for Krasnoyarsk region population basing on the data provided by social and hygienic monitoring

Data and methods. To analyze primary morbidity dynamics for Krasnoyarsk region population we used data taken from the state statistic observation form No. 12 "Information on number of diseases registered in patients living in the area of a medical organization service" provided by Krasnoyarsk Public Health Ministry. The data comprised children, teenagers and adult population groups; the total population number in Krasnoyarsk region amounted to $2,866,490$ people (on the 1 st of January, 2015) and it was taken as per each of
55 city districts and municipal districts separately over 2005-2015 as well.

We analyzed long-term (2005-2015) primary morbidity dynamics for Krasnoyarsk region population; we calculated morbidity forecast for diseases categories taken as per International Diseases Classification, Revision $\mathrm{X}$, taking all the revealed trends and correlation with average long-term indexes into account [6]. We also calculated and assessed population health "risk" degree in conformity with "Integral population health assessment on territories" guidelines [5]. Integral health index, in addition to comparison between territories, has a criterion score which corresponds to different degree of population health risk in territories: minimal risk value is less than 0.312 ; moderate risk value amounts to 0.313 0.500 ; increased risk value is $0.501-0.688$; and if the value is higher than 0.689 risk is high.

We assessed authentic discrepancies between compared average values depending on t-criterion value (Student's t-criterion). We used MS Excel to analyze and statistically process the information.

Results and discussion. Primary morbidity (with a diagnosis made for the first time) among Krasnoyarsk region population amounted to 785.9 cases per 1,000 people in 2015 which was authentically lower by $2 \%$ than in 2014. Respiratory organs diseases occupy the 1 st place in primary morbidity structure, both in the region as a whole and as per different age groups (children, teenagers and adults) $(35.2 \%, 59.5 \%, 42.8 \%, 18.7 \%$ correspondingly). The 2nd place belongs to injuries, intoxications and some other consequences of external causes $(13.7 \%, 6.4 \%, 14.6 \%$, and $18.5 \%$ correspondingly). The 3 rd place among children and teenagers belongs to skin and subcutaneous tissue diseases $(5.2 \%$ in the region as a whole, $4.9 \%$ and $5.6 \%$ correspondingly); urogenital system diseases have the same place among adults (7.4\% in the region as a whole, $11.2 \%$ correspondingly).

Table 1 contains primary morbidity indexes for Krasnoyarsk region population taken in dynamics over 2011-2015 and 
compared with average country morbidity indexes.

As per data given in Table 1, primary morbidity indexes for Krasnoyarsk region population were higher than average country indexes in 2011-2014 for such diseases as neoplasms, nervous system diseases, circulatory and digestive system diseases, musculoskeletal system and connective tissue diseases, endocrine system and urogenital system diseases, eye and ear diseases, injuries, and intoxications. Morbidity for respiratory organs diseases, blood and blood-making organs diseases, skin and subcutaneous tissue diseases in Kras- noyarsk region is lower than in the Russian Federation on average. There are no authentic discrepancies in the morbidity levels for digestive organs diseases, skin and subcutaneous tissue diseases and congenital abnormalities (malformations) between Krasnoyarsk region and the Russian Federation as a whole.

Dynamics of primary morbidity indexes for Krasnoyarsk region population in 20102014 is characterized with an authentic trend showing both growth and reduction and it coincides with the overall trends of changes in the whole country indexes.

Table 1

Primary morbidity dynamics in Krasnoyarsk region and in the Russian Federation, number of cases per 1000 people, \%o

\begin{tabular}{|c|c|c|c|c|c|c|}
\hline \multirow[b]{2}{*}{ Disease category, territory } & \multicolumn{5}{|c|}{ year } & \multirow{2}{*}{$\begin{array}{l}\text { Annual average } \\
\text { growth rate }(\boldsymbol{\Delta}), \text { de- } \\
\text { crease rate }(\boldsymbol{\nabla}), \%\end{array}$} \\
\hline & $2011 *$ & $2012 *$ & 2013 & 2014 & 2015 & \\
\hline \multicolumn{7}{|c|}{ Registered diseases, total } \\
\hline Russian Federation $^{1}$ & 796.9 & 793.9 & 799.4 & 787.1 & $\mathrm{n} / \mathrm{a}$ & $\Delta 0.2$ \\
\hline Krasnoyarsk region & 832.62 & 829.3 & 808.8 & 805.7 & 785.9 & $\boldsymbol{\nabla} 1.4$ \\
\hline \multicolumn{7}{|c|}{ Some infectious and parasitic diseases } \\
\hline Russian Federation $^{1}$ & 32.4 & 32.1 & 30.9 & 30.8 & $\mathrm{n} / \mathrm{a}$ & $\nabla 1.7$ \\
\hline Krasnoyarsk region & 30.73 & 36.22 & 34.6 & 34.9 & 30.8 & $\nabla 0.3$ \\
\hline \multicolumn{7}{|c|}{ Neoplasms } \\
\hline Russian Federation $^{1}$ & 32.4 & 32.1 & 30.9 & 30.8 & $\mathrm{n} / \mathrm{a}$ & $\nabla 1.7$ \\
\hline Krasnoyarsk region & 30.73 & 36.22 & 34.6 & 34.9 & 30.8 & $\boldsymbol{\nabla} 0.3$ \\
\hline \multicolumn{7}{|c|}{$\begin{array}{l}\text { Blood and blood-making organs diseases } \\
\text { and certain disorders involving immune mechanism }\end{array}$} \\
\hline Russian Federation $^{1}$ & 4.7 & 4.7 & 4.7 & 4.7 & $\mathrm{n} / \mathrm{a}$ & $\nabla 0.8$ \\
\hline Krasnoyarsk region & 4.26 & 4.08 & 3.8 & 3.9 & 3.8 & $\boldsymbol{\nabla} 2.6$ \\
\hline \multicolumn{7}{|c|}{ Endocrine system diseases, digestive disorders and metabolic disorders } \\
\hline Russian Federation $^{1}$ & 10.3 & 10.6 & 10.6 & 11.2 & $\mathrm{n} / \mathrm{a}$ & $\mathbf{\Delta} 2.2$ \\
\hline Krasnoyarsk region & 12.23 & 12.18 & 11.1 & 11.7 & 13.4 & $\Delta 1.6$ \\
\hline \multicolumn{7}{|c|}{ Nervous system diseases } \\
\hline Russian Federation $^{1}$ & 16.5 & 16.3 & 16.5 & 16.2 & $\mathrm{n} / \mathrm{a}$ & $\boldsymbol{\nabla} 0.2$ \\
\hline Krasnoyarsk region & 20.01 & 18.24 & 19.0 & 19.1 & 16.9 & $\boldsymbol{\nabla} 2.9$ \\
\hline \multicolumn{7}{|c|}{ Eye and its accessory apparatus diseases } \\
\hline Russian Federation $^{1}$ & 33.3 & 35.2 & 35.0 & 34.7 & $\mathrm{n} / \mathrm{a}$ & $\Delta 1.5$ \\
\hline Krasnoyarsk region & 47.26 & 45.9 & 44.0 & 43.6 & 41.5 & $\nabla 3.1$ \\
\hline \multicolumn{7}{|c|}{ Ear and mastoid diseases } \\
\hline Russian Federation $^{1}$ & 27.8 & 28.2 & 28.0 & 27.7 & $\mathrm{n} / \mathrm{a}$ & $\Delta 0.5$ \\
\hline Krasnoyarsk region & 30.28 & 31.35 & 29.8 & 29.9 & 27.7 & $\nabla 2.2$ \\
\hline \multicolumn{7}{|c|}{ Circulatory system diseases } \\
\hline Russian Federation $^{1}$ & 28.2 & 26.6 & 29.9 & 28.8 & $\mathrm{n} / \mathrm{a}$ & $\mathbf{\Delta} 1.3$ \\
\hline
\end{tabular}




\begin{tabular}{|c|c|c|c|c|c|c|}
\hline \multirow[b]{2}{*}{ Disease category, territory } & \multicolumn{5}{|c|}{ year } & \multirow{2}{*}{$\begin{array}{c}\text { Annual average } \\
\text { growth rate }(\boldsymbol{\Delta}), \text { de- } \\
\text { crease rate }(\boldsymbol{\nabla}), \%\end{array}$} \\
\hline & $2011 *$ & $2012 *$ & 2013 & 2014 & 2015 & \\
\hline Krasnoyarsk region & 31.35 & 32.78 & 34.4 & 32.9 & 33.9 & $\Delta 0.5$ \\
\hline \multicolumn{7}{|c|}{ Respiratory organs diseases } \\
\hline Russian Federation $^{1}$ & 338.8 & 330.9 & 338.4 & 333.4 & $\mathrm{n} / \mathrm{a}$ & $\Delta 0.5$ \\
\hline Krasnoyarsk region & 311.29 & 295.89 & 295.3 & 286.1 & 276.5 & $\nabla 2.7$ \\
\hline \multicolumn{7}{|c|}{ Digestive organs diseases } \\
\hline Russian Federation $^{1}$ & 34.8 & 34.8 & 35.2 & 36.6 & $\mathrm{n} / \mathrm{a}$ & $\mathbf{\Delta} 2.0$ \\
\hline Krasnoyarsk region & 36.6 & 36.6 & 35.3 & 38.7 & 37.1 & r 0.2 \\
\hline \multicolumn{7}{|c|}{ Skin and subcutaneous tissue diseases } \\
\hline Russian Federation $^{1}$ & 48.0 & 48.0 & 47.0 & 46.3 & $\mathrm{n} / \mathrm{a}$ & $\boldsymbol{\nabla} 0.7$ \\
\hline Krasnoyarsk region & 43.77 & 43.77 & 42.3 & 41.5 & 40.8 & r 0.1 \\
\hline \multicolumn{7}{|c|}{ Musculoskeletal system and connective tissue diseases } \\
\hline Russian Federation $^{1}$ & 33.6 & 33.2 & 32.3 & 31.8 & $\mathrm{n} / \mathrm{a}$ & $\boldsymbol{\nabla} 1.4$ \\
\hline Krasnoyarsk region & 43.68 & 43.68 & 40.3 & 41.7 & 41.1 & $\nabla 1.9$ \\
\hline \multicolumn{7}{|c|}{ Urogenital system diseases } \\
\hline Russian Federation $^{1}$ & 49.3 & 49.6 & 49.8 & 49.0 & $\mathrm{n} / \mathrm{a}$ & $\Delta 0.6$ \\
\hline Krasnoyarsk region & 55.8 & 57.33 & 54.4 & 54.6 & 58.5 & $\Delta 0.5$ \\
\hline \multicolumn{7}{|c|}{ Congenital abnormalities (malformations) } \\
\hline Russian Federation $^{1}$ & 2.1 & 2.1 & 2.1 & 2.1 & $\mathrm{n} / \mathrm{a}$ & $\Delta \boldsymbol{\nabla} 0.0$ \\
\hline Krasnoyarsk region & 2.12 & 1.78 & 1.9 & 1.8 & 2.1 & S 0.6 \\
\hline \multicolumn{7}{|c|}{ Injuries. intoxications and some other consequences of external causes } \\
\hline Russian Federation $^{1}$ & 92.80 & 93.8 & 92.6 & 90.2 & $\mathrm{n} / \mathrm{a}$ & $\nabla 0.3$ \\
\hline Krasnoyarsk region & 111.47 & 113.16 & 107.3 & 109.6 & 108.0 & $\nabla 0.9$ \\
\hline
\end{tabular}

Note: * - data taken from EMISS of Federal Service of State Statistics ; $r$ - statistically not authentic trend; ${ }^{1}$ - annual average growth/decrease rate is calculated for the period of 2010-2014.

Thus, morbidity level for blood and blood-making organs diseases tended to decrease with average annual decrease rate equal to $5.4 \%$ in Krasnoyarsk region and $0.8 \%$ in the Russian Federation; for nervous system diseases, $2.1 \%$ and $0.2 \%$ correspondingly; for musculoskeletal system diseases, $1.9 \%$ and $1.4 \%$ correspondingly. Morbidity levels for neoplasms and circulatory system diseases tended to grow both in Krasnoyarsk region and in the Russian Federation in 2010-2014 and annual average increase rate in Krasnoyarsk region was higher than in the $\mathrm{RF}$ as a whole being equal to $5.8 \%$ and $2.6 \%$ in Krasnoyarsk region correspondingly and $1.7 \%$ and $1.3 \%$ in the RF correspondingly.

Overall primary morbidity continued to decrease in Krasnoyarsk region in 2015 and annual average decrease rate over 2011-2015 was equal to $1.4 \%$. Statistically authentic decrease in primary morbidity was detected for the following diseases: blood diseases (annual average decrease rate was equal to $2.6 \%$ ); nervous system diseases (annual average decrease rate was equal to $2.8 \%$ ); respiratory organs diseases (annual average decrease rate was 2.7\%); eye and its accessory apparatus diseases (annual average decrease rate was equal to $3.0 \%$ ); musculoskeletal system diseases (annual average decrease rate was equal to $1.9 \%$ ); ear diseases (annual average decrease rate was equal to $2.2 \%$ ); the data are shown in Figures 1, 2, and 3. Primary morbidity for certain diseases categories tends to grow; it is true for neoplasms (annual average growth rate is equal to $4.3 \%$ ); endocrine system diseases (annual average growth rate is equal to $1.5 \%$ ); circulatory system diseases (annual average growth rate is equal to $0.5 \%$ ); urogenital system diseases (annual average growth is equal to $0.5 \%$ ); the trend is shown in Figure 4. (The y-axis description to the left: cases per 1000 people a year). 


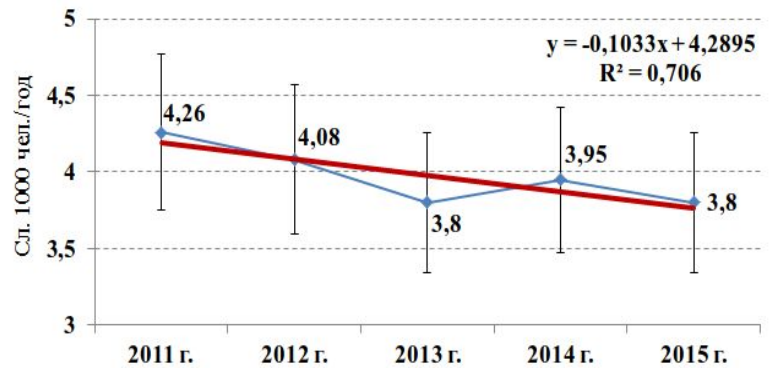

Figure 1. Decrease trend of primary morbidity for blood and blood-making organs diseases in Krasnoyarsk region, 2011-2015 $(\%$ \%0).

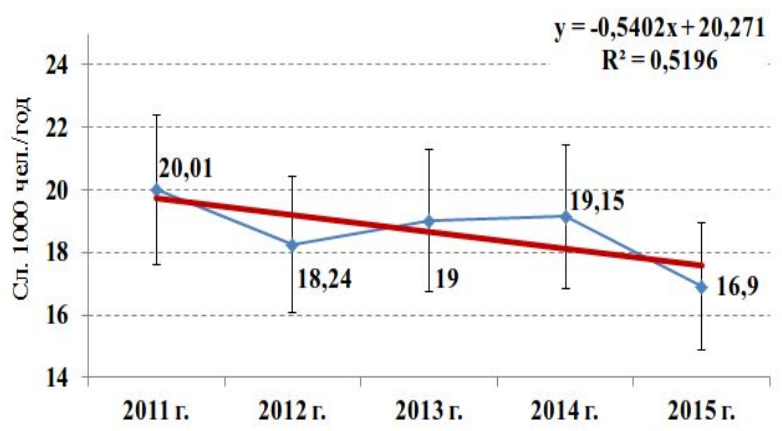

Figure_2. Decrease trend of primary morbidity for nervous system diseases in Krasnoyarsk region, 2011-2015 $(\%)$

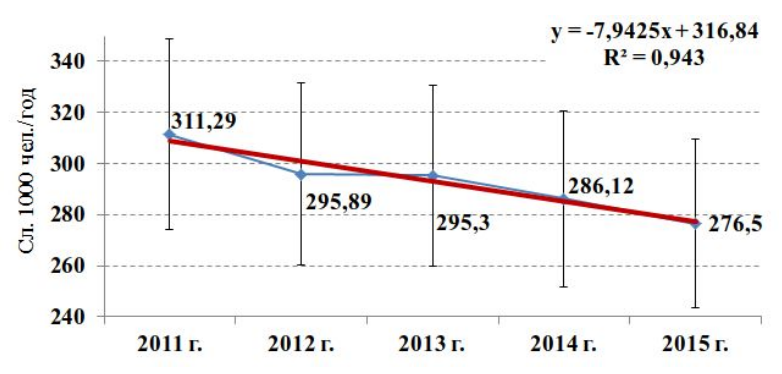

Figure_3. Decrease trend of primary morbidity for respiratory organs diseases in Krasnoyarsk region, 2011-2015 $(\% 0)$

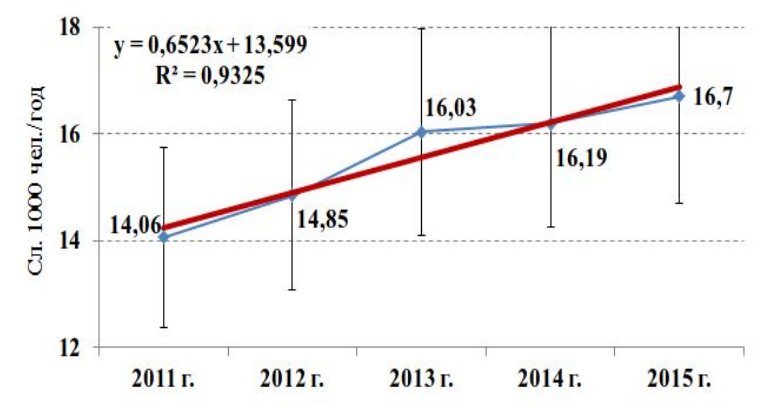

Figure 4. Increase trend of primary morbidity for neoplasms in Krasnoyarsk region, 2011$2015(\%)$

Table 2

Dynamics and forecast of primary morbidity indexes for Krasnoyarsk region population ( $\mathrm{p}<0.005)$

\begin{tabular}{|c|c|c|c|c|c|}
\hline \multirow{2}{*}{ Disease category } & \multirow{2}{*}{$\begin{array}{c}\text { Annual average } \\
\text { growth rate }(\boldsymbol{\Lambda}), \\
\text { decrease rate } \\
(\boldsymbol{\nabla}), \%\end{array}$} & \multicolumn{2}{|c|}{$\begin{array}{l}\text { Forecast, } \\
2016 .\end{array}$} & \multicolumn{2}{|c|}{$\begin{array}{l}\text { forecast, } \\
2017 .\end{array}$} \\
\hline & & Index & $\Delta$ & Index & $\Delta$ \\
\hline Total number of diseases & $\mathbf{\Delta} 0.2$ & 824.0 & 0.44 & 825.6 & 0.44 \\
\hline Some infectious and parasitic diseases & $\boldsymbol{\nabla} 2.9$ & 30.1 & 0.2 & 29.1 & 0.19 \\
\hline Neoplasms & $\Delta 3.98$ & 17.3 & 0.15 & 17.8 & 0.15 \\
\hline $\begin{array}{l}\text { Blood and blood-making organs diseases and some dis- } \\
\text { orders involving immune mechanism }\end{array}$ & $\boldsymbol{\nabla} 1.1$ & 3.97 & 0.07 & 3.92 & 0.07 \\
\hline $\begin{array}{l}\text { Endocrine system diseases, digestive disorders and met- } \\
\text { abolic disorders }\end{array}$ & $\mathbf{\Delta} 2.3$ & 12.9 & 0.13 & 13.2 & 0.13 \\
\hline Nervous system diseases & $\mathbf{\Delta} 1.2$ & 19.7 & 0.16 & 19.9 & 0.16 \\
\hline Eye and its accessory apparatus diseases & $\boldsymbol{\nabla} 0.3$ & 44.4 & 0.24 & 44.3 & 0.24 \\
\hline Ear and mastoid diseases & $\mathbf{\Delta} 0.8$ & 30.6 & 0.2 & 30.8 & 0.2 \\
\hline Circulatory system diseases & $\mathbf{\Delta} 0.5$ & 34.7 & 0.2 & 34.9 & 0.2 \\
\hline Respiratory organs diseases & $\mathbf{\Delta} 0.8$ & 301.3 & 0.53 & 303.6 & 0.53 \\
\hline Digestive organs diseases & $\mathbf{\Delta} 0.3$ & 38.5 & 0.22 & 38.6 & 0.22 \\
\hline Skin and subcutaneous system diseases & $\nabla 3.7$ & 36.5 & 0.22 & 34.7 & 0.21 \\
\hline Musculoskeletal system and connective tissue diseases & $\Delta 0.5$ & 43.4 & 0.23 & 43.6 & 0.24 \\
\hline Urogenital system diseases & $\mathbf{\Delta} 1.5$ & 59.6 & 0.27 & 60.5 & 0.28 \\
\hline $\begin{array}{l}\text { Injuries, intoxications and some other external causes } \\
\text { consequences }\end{array}$ & $\boldsymbol{\nabla} 0.2$ & 109.1 & 0.36 & 108.9 & 0.36 \\
\hline
\end{tabular}

Note: $\Delta$ is confidence interval 
According to forecast calculations performed on the basis of long-term observations (2005-2015) of primary morbidity in Krasnoyarsk region we can expect possible growth in morbidity indexes for all diseases categories by $2016-2017$, except blood diseases, bloodmaking organs diseases and certain disorders involving immune mechanism, skin and subcutaneous tissue diseases, eye and its accessory apparatus diseases, infectious and parasitic diseases, injuries and intoxications (Table 2).

As we looked into territorial distribution of population morbidity indexes we detected that primary morbidity indexes for main diseases categories in some Krasnoyarsk region territories are authentically 1.5 and more times higher than in the region as a whole (Student's $\mathrm{t}$ criterion is $>2$ ), and such territories can be considered "risk" ones. Risk territories where primary morbidity levels exceed average regional one include Idrinskiy district and Shushenskiy district (12 diseases categories); Norilsk, Evenkiyskiy district, Krasnoyarsk, Nazarovo (11 diseases categories); Achinsk and Dzerzhinskiy district (10 diseases categories); Minusinsk, Sharyipovo and Taseevskiy district (9 diseases categories); Divnogorsk, Lesosibirsk, Balakhtinsliy district, Bolsheuluyskiy district, Severo-Eniseyskiy district, Uzhurskiy district (8 diseases categories); Bogotolskiy district (7 diseases categories); $\mathrm{Bi}-$ rilyusskiy district (6 diseases categories); $\mathrm{Ku}$ raginsliy district (5 diseases categories).

The accomplished analysis of population health indexes in 55 urban districts and municipal districts of Krasnoyarsk region revealed that diseases caused by various adverse environmental factors prevail in most territories. We also determined risk territories where levels of primary morbidity caused by environmental factors effects were authentically 1.5 and more times higher than average regional levels. 15 territories were included into risk category as per neoplasms; 19 territories, as per blood and blood-making organs diseases; 16 territories, as per endocrine system diseases; 13 territories, as per nervous system diseases; 25 territories, as per circulatory system diseases; 21 territories, as per respiratory organs diseases; 11 territories, as per malignant neoplasms (Table 3).

Table 3

The list of Krasnoyarsk region territories where level of primary morbidity caused by environmental factors effects was authentically higher than average regional level, 2015

\begin{tabular}{|c|c|}
\hline Disease category & City or municipal district \\
\hline Neoplasms & $\begin{array}{l}\text { Achinsk, Divnogorsk, Krasnoyarsk, Minusinsk, Nazarovo, Norilsk, Sharyipovo, } \\
\text { Bolsheuluyskiy district, Eniseyskiy district, Idrinskiy district, Kezhemskiy district, } \\
\text { Kuraginskiy district, Nizhneingashskiy district, Severo-Eniseyskiy district, Shushen- } \\
\text { skiy district }\end{array}$ \\
\hline $\begin{array}{l}\text { Blood and blood-making } \\
\text { organs diseases and cer- } \\
\text { tain disorders involving } \\
\text { immune mechanism }\end{array}$ & $\begin{array}{l}\text { Lesosibirsk, Minusinsk, Sharyipovo, Balakhtinskiy district, Birilyusskiy district, } \\
\text { Bogotolskiy district, Dzerzhinskiy district, Eniseyskiy district, Ermakovskiy district, } \\
\text { Idrinskiy district, Kazachinskiy district, Kozulskiy district, Kuraginskiy district, } \\
\text { Novosyolovskiy district, Taseevskiy district, Turukhanskiy district, Uzhurskiy dis- } \\
\text { trict, Shushenskiy district, Evenkiyskiy district }\end{array}$ \\
\hline $\begin{array}{l}\text { Endocrine system diseas- } \\
\text { es, digestive disorders and } \\
\text { metabolic disorders }\end{array}$ & $\begin{array}{l}\text { Divnogorsk, Krasnoyarsk, Minusinsk, Sharyipovo, Berezovskiy district, Bogotol- } \\
\text { skiy district, Dzerzhinskiy district, Idrinskiy district, Irbeyskiy district, Karatuzskiy } \\
\text { district, Kozulskiy district, Novosyolovskiy district, Pirovskiy district, Sayanskiy } \\
\text { district, Shushenskiy district, Evenkiyskiy district }\end{array}$ \\
\hline Nerve & $\begin{array}{l}\text { Achinsk, Krasnoyarsk, Krasnoyarsk, Nazarovo, Birilyusskiy district, Dzerzhinskiy } \\
\text { district, Idrinskiy district, Krasnoturanskiy district, Kuraginskiy district, Taseevskiy } \\
\text { district, Uzhurskiy district, Shushenskiy district, Evenkiyskiy district }\end{array}$ \\
\hline $\begin{array}{l}\text { Circulatory system dis- } \\
\text { eases }\end{array}$ & $\begin{array}{l}\text { Krasnoyarsk, Lesosibirsk, Minusinsk, Nazarovo, Norilsk, Balakhtinskiy district, } \\
\text { Berezovskiy district, Birilyusskiy district, Bogotolskiy district, Bolshemurtinskiy } \\
\text { district, Bolsheuluyskiy district, Dzerzhinskiy district, Ermakovskiy district, Idrin- } \\
\text { skiy district, Karatuzskiy district, Krasnoturanskiy district, Kuraginskiy district, } \\
\text { Manskiy district, Motyiginskiy district, Severo-Eniseyskiy district, Taseevskiy dis- } \\
\text { trict, Turukhanskiy district, Uzhurskiy district, Shushenskiy district, Evenkiyskiy }\end{array}$ \\
\hline
\end{tabular}


Peculiarities of territorial distribution and dynamics in rates of population noncommunicable diseases in...

\begin{tabular}{|l|l|}
\hline \multicolumn{1}{|c|}{ Disease category } & \multicolumn{1}{c|}{ City or municipal district } \\
\hline \multirow{3}{*}{$\begin{array}{l}\text { Respiratory organs dis- } \\
\text { eases }\end{array}$} & district \\
& $\begin{array}{l}\text { Achinsk, Borodino, Divnogorsk, Kansk, Lesosibirsk, Nazarovo, Norilsk, Sharyipo- } \\
\text { vo, Balakhtinskiy district, Boguchanskiy district, Partizanskiy district, Ryibinskiy } \\
\text { district, Severo-Eniseyskiy district, Sukhobuzimskiy district, Taymyirskiy district, } \\
\text { Dolgano-Nanatskiy district, Taseevskiy district, Turukhanskiy district, Tyukhtetskiy } \\
\text { district, Uzhurskiy district, Shushenskiy district, Evenkiyskiy district }\end{array}$ \\
\hline \multirow{3}{*}{ Malignant neoplasms } & $\begin{array}{l}\text { Achinsk and Achinskiy district, Borodino, Krasnoyarsk, Lesosibirsk, Minusinsk and } \\
\text { Minusinskiy district, Sharyipovo and Sharyipovskiy district, Berezovskiy district, } \\
\text { Ermakovskiy district, Novosyolovskiy district, Sukhobuzimskiy district, Shushen- } \\
\text { skiy district }\end{array}$ \\
\hline
\end{tabular}

Table 4

Population number who live in Krasnoyarsk region territories with different health risk degree in 2014-2016

\begin{tabular}{|l|c|c|c|c|c|c|}
\hline \multirow{2}{*}{ Risk degree } & \multicolumn{2}{|c|}{2014} & \multicolumn{2}{c|}{2015} & \multicolumn{2}{c|}{2016} \\
\cline { 2 - 7 } & $\begin{array}{c}\text { Population } \\
\text { number }\end{array}$ & $\begin{array}{c}\text { Share of } \\
\text { total popu- } \\
\text { lation, \% }\end{array}$ & $\begin{array}{c}\text { Population } \\
\text { number }\end{array}$ & $\begin{array}{c}\text { Share of } \\
\text { total popu- } \\
\text { lation, \% }\end{array}$ & $\begin{array}{c}\text { Population } \\
\text { number }\end{array}$ & $\begin{array}{c}\text { Share of } \\
\text { total popu- } \\
\text { lation, \% }\end{array}$ \\
\hline Minimal & 238339 & 8.9 & 453004 & 16.8 & 490395 & 18.2 \\
\hline Moderate & 638512 & 23.8 & 444119 & 16.5 & 416778 & 15.4 \\
\hline Increased & 1734978 & 64.6 & 1778952 & 66.1 & 1733814 & 64.2 \\
\hline High & 72790 & 2.7 & 15425 & 0.6 & 59198 & 2.2 \\
\hline
\end{tabular}

The analysis shows that diseases registration frequency is the highest in such industrial cities as Achinsk, Krasnoyarsk, Lesosibirsk, Minusinsk, Nazarovo, and Norilsk, where population is influenced by apparent chemical load.

We assessed and compared degree of population health risk in urban districts and municipal districts of Krasnoyarsk region basing on the integral index calculation [16] with the use of the data collected over long-term observation period. The results show that "increased" degree of population health risk in 2015 was detected in 21 territories where $66.1 \%$ of Krasnoyarsk region population live. These territories include Achinsk, Divnogorsk, Krasnoyarsk, Norilsk, Lesosibirsk, Minusinsk, Nazarovo, Sharyipovo, Achinskiy district, Achinskiy district, Bogotolskiy district, Bolsheuluyskiy district, Dzerzhinskiy district, Idrinskiy district, Krasnoturansliy district, Minusinskiy district, Nazarovskiy district, Taseevskiy district, Uzhurskiy district, Sharyipovskiy district, Shushenskiy district. In 2015 Evenkiyskiy district was included into "high population health risk" category (in 2014 this category included Sharyipovo, Birilyuss- kiy district and Evenkiyskiy district). According to forecasts, in 2016 we expect to see decrease in specific weight of the region population who are included into "moderate health risk" and "increased health risk" categories and growth in population who are included into "high health risk" and " minimal health risk" categories (Table 4).

So, population health in Krasnoyarsk region expressed in terms of primary morbidity indexes is characterized with high levels of non-infectious morbidity, including one caused by environmental factors; these levels are higher than average country ones.

Population morbidity indexes for various diseases categories have been changing in both directions over the last few years in Krasnoyarsk region, growing and falling; such trends are characteristic both for the Russian Federation as a whole and Krasnoyarsk region, but decrease and increase rates for several indexes in Krasnoyarsk region are higher. As forecast calculations based on the data received from long-term observations show, by 2016-2017 we can expect to see possible growth in primary morbidity of Krasnoyarsk region population. 
Territorial distribution reveals that primary morbidity levels in significant number of cities and districts in Krasnoyarsk region are statistically authentically higher than average regional levels; morbidity registration frequency is the highest in industrial cities where chemical load is apparent; such cities are Achinck, Krasnoyarsk, Lesosibirsk, Minusinsk, Nazarovo, and Norilsk, including areas adjacent to them, where $66.1 \%$ of Krasnoyarsk region population live.
Conclusion: Population health in Krasnoyarsk region is characterized with high indexes of non-infectious morbidity related to various risk factors influence. The accomplished comparative assessment of population health gives health characteristics on territorial-population level; it helps to determine problem territories where measures aimed at health improvement and morbidity prevention are to be implemented.

\section{References}

1. Akatova A.A., Sharova L.V., Aminova A.A. Vlijanie tehnogennyh faktorov okruzhajushhej sredy na sostojanie zdorov'e detej [The impact of man-made environmental factors on children's health]. In: Adaptivnaja fizicheskaja kul'tura, sport i zdorov'e: integracija nauki i praktiki: sb. tr. II Vseros. nauch.-prakt. konf. Ufa, 2010, part 1, pp. 19-21 (in Russian).

2. Dautov F.F., Hakimova R.F., Jusupova N.Z. Vlijanie zagrjaznenij atmosfernogo vozduha na allergicheskuju zabolevaemost' detej v krupnom promyshlennom gorode [Impact of ambient air pollution on allergic morbidity in children in a large industrial town]. Gigiena $i$ sanitarija, 2007, no. 2, pp. 10-12 (in Russian).

3. Doklad o sostojanii zdravoohranenija v mire 2010 goda [Report on world health for the year 2010]. Vsemirnaja organizacija zdravoohranenija, 2010, 126 p. Available at: www.un.org/ru/development/surveys/docs/whr2010.pdf (12.10.2016) (in Russian).

4. Zaitseva N.V., May I.V., Balashov S.Ju. Mediko-biologicheskie pokazateli sostojanija zdorov'ja naselenija $\mathrm{v}$ uslovijah kompleksnogo prirodno-tehnogennogo zagrjaznenija sredy obitanija [Medical and biologic parameters of the population health state in conditions of inhabitancy complex naturaltechnogenic pollution]. Izvestija Samarskogo nauchnogo centra Rossijskoj Akademii nauk, 2009, vol. 11, no. 1-6, pp. 1144-1148 (in Russian).

5. Integral'naja ocenka sostojanija zdorov'ja naselenija na territorijah: metodicheskie ukazanija (utv. Goskomsanjepidnadzorom RF 25.09.1995) [Integral assessment of health status in the territories: guidelines (approved by the State Committee of the Russian Federation on 25.09.1995)]. Moscow, Federal'nyj centr Gossanjepidnadzora Publ., 1995, 42 p. (in Russian).

6. Marchenko B.I. Zdorov'e na populjacionnom urovne: statisticheskie metody issledovanija: rukovodstvo dlja vrachej [Health at the population level: statistical methods: a guide for physicians]. Taganrog, Sfinks Publ., 1997, 432 p. (in Russian).

7. Merkulova N.A. Prognozirovanie zabolevaemosti detskogo naselenija pri izmenenii zagrjaznenija atmosfernogo vozduha promyshlennogo goroda (na primere g. Vladikavkaza): diss. kand. med. nauk [Forecasting of child morbidity in changing atmospheric pollution of an industrial city (on an example of Vladikavkaz) Thesis of Candidate of Medicine]. Kazan', 2011,176 p.

8. Popova A.Ju., Zaitseva N.V., May I.V., Kiryanov D.A. Metodicheskie podhody k raschetu fakticheskih i predotvrashhennyh mediko-demograficheskih i jekonomicheskih poter', associirovannyh s negativnym vozdejstviem faktorov sredy obitanija [Methodological approaches to the calculation of actual and prevented as a result of the control and supervisory activities, medical-demographic and economic 95 losses, associated with the negative impact of environmental factors]. Gigiena $i$ sanitarija, 2015, vol. 94, no. 7, pp. 95-99 (in Russian).

9. Onishchenko G.G. Itogi i perspektivy obespechenija sanitarno-jepidemiologicheskogo blagopoluchija naselenija Rossijskoj Federacii [Results and prospects of the guarantee of sanitaryepidemiological welfare of population of the Russian Federation]. Gigiena $i$ sanitarija, 2012, no. 4, pp. 4-12 (in Russian). 
10. Verevina M.L., Rusakov N.V., Zhukova T.V., Gruzdeva O.A. Ocenka zabolevaemosti naselenija $\mathrm{v}$ zavisimosti ot uslovij prozhivanija [Estimation of morbidity rates in relation to living conditions]. Gigiena i sanitarija, 2010, no. 3, pp. 21-25 (in Russian).

11. Popova A.Ju. Strategicheskie prioritety Rossijskoj Federacii v oblasti jekologii s pozicii sohranenija zdorov'ja nacii [Strategic priorities of the Russian Federation in the field of ecology from the position of preservation of health of the nation]. Zdorov'e naselenija i sreda obitanija, 2014, vol. 251, no. 2, pp. 4-7 (in Russian).

12. Potapov A.I., Novichkova N.I., Chistjakova T.V., Parhomenko V.V. Profilaktika i reabilitacija - jeffektivnye napravlenija povyshenija urovnja zdorov'ja naselenija [Prevention and rehabilitation are effective areas to improve population health]. Zdravoohranenie Rossijskoj Federacii, 2012, no. 1, pp. 35 (in Russian).

13. Sadovnikova Ju.M. Sravnitel'nye pokazateli zabolevaemosti detej doshkol'nogo vozrasta iz jekologicheski razlichnyh gorodskih mikrorajonov [Comparative morbidity of preschool children from ecologically different urban neighborhoods]. Vestnik orenburgskogo gosudarstvennogo universiteta, 2009, vol. 106, no. 12, pp. 56-60 (in Russian).

14. Chamkina T.I. Vlijanie otraslej promyshlennosti na sostojanie okruzhajushhej sredy Kuzbassa [The impact of industries on Kuzbass environment]. Gornyj informacionno-analiticheskij bjulleten' (nauchno-tehnicheskij zhurnal), 2008, no. 8, pp. 268-279 (in Russian).

15. Shur P.Z., Zubarev A.Ju., Sharifov A.T. Metodicheskie podhody k razrabotke kriteriev dlja kolichestvennoj ocenki nekancerogennogo riska faktorov sredy obitanija po rezul'tatam jepidemiologicheskih issledovanij [Methodological approaches to the development of criteria to quantify the non-cancer risk of environmental factors on the results of epidemiological studies]. Profilakticheskaja medicina v Rossii: istoki i sovremennost': sb. mater. Vseros. konf. s mezhdunar. uchastiem, posv. 140-letiju obrazovanija pervoj gigienicheskoj kafedry v Rossii [Preventive medicine in Russia: the origins and the present: the collection of materials of the All-Russian conference with international participation]. Kazan', Kazan. gos. med. un-ta Publ., 2009, vol. 2, pp. 105-106 (in Russian).

16. Arutjunjan R.V., Vorob'eva L.M., Panchenko S.V., Pechkurova K.A., Novikov S.M., Shashina T.A., Dodina N.S., Gorjaev D.V., Tihonova I.V., Kurkatov S.V., Skudarnov S.E., Ivanova O.Ju. Jekologicheskaja bezopasnost' Krasnojarskogo kraja s pozicii analiza riska dlja zdorov'ja naselenija [Environmental safety assessment of Krasnoyarsk Krai based on a public health risk analysis]. Atomnaja jenergija, 2015, vol. 118, no. 2, pp. 113-117 (in Russian).

17. Ulanova T.S., Nurislamova T.V., Popova N.A., Chin'ko T.V. Jekologo-gigienicheskaja harakteristika sredy obitanija i ocenka zdorov'ja detej v uslovijah tehnogennoj nagruzki [Ecological and hygienic characteristics of the environment and evaluation of children's health in the conditions of anthropogenic impact]. Nauchnye osnovy i mediko-profilakti-cheskie tehnologii obespechenija sanitarnojepidemiologicheskogo blagopoluchija naselenija: mater. Vseros. nauch.-prakt. konf. [Scientific basis of medical and preventive technology of sanitary and epidemiological welfare of the population: Proceedings of the scientific-practical conference]. In: chl.-korr. RAMN, d-r med. nauk, prof. N.V. Zaitseva ed. Perm', 2009, pp. 45-51 (in Russian).

Goryaev D.V., Tikhonova I.V. Peculiarities of territorial distribution and dynamics in rates of population noncommunicable diseases in the Krasnoyarsk region associated with the influence of environmental risk factors. Health Risk Analysis, 2016, no. 4, pp. 49-57. DOI: 10.21668/health.risk/2016.4.07.eng

Received: 12.09 .2016

Accepted: 12.12 .2016

Published: 30.12.2016 